\title{
Theoretical Analysis of Optical Spatial Multiple Pulse Position Modulation
}

\author{
Thai-Chien Bui, IEEE Student Member, Mauro Biagi, IEEE Senior Member, Suwit Kiravittaya, IEEE Member
}

\begin{abstract}
This paper proposes an advanced multi-pulse multilight emitting diodes (LEDs) modulation technique to improve the efficiency for indoor visible light communication systems. The technique is referred to as spatial multiple pulse position modulation (SMPPM), and it is developed by combining a high spectral efficiency space shift keying (SSK) with a high energy efficiency multiple pulse position modulation (MPPM). During a symbol transmission, multiple active pulse slots and active LED indices are utilized as two degrees of freedom to modulate information which in turn provides the balance between the complexity, achievable spectral efficiency and energy efficiency. The analytical expression for symbol error rate (SER) of SMPPM in a multiple input multiple output system with multi-path propagation from reflection is derived theoretically using union bound technique and validated by means of Monte-Carlo simulations. Error performance of SMPPM is then evaluated extensively for different transceiver parameters and pulse configurations. Error rate distribution in a typical indoor workspace is plotted and analyzed. The SMPPM system achieves significantly higher spectral efficiency with respect to the conventional MPPM, SSK and spatial pulse position modulation (SPPM).
\end{abstract}

Index Terms-Visible light communication, pulse modulation, spectral efficiency.

\section{INTRODUCTION}

In visible light communication (VLC), multiple noncoherent light emitting diodes (LEDs) are normally used for both functionalities of illumination and data transmission [1], [2]. The intensity and wavelength of light emitted from the LEDs are the most widely used degrees to modulate information due to the fast-switching and the wide visible light spectrum of recent advanced LEDs. Some conventional techniques that are capable of modulating data into intensity of light are on-off keying (OOK), unipolar pulse amplitude modulation (PAM), pulse position modulation (PPM) and orthogonal frequency division multiplexing (OFDM) [3]. Besides the intensity of light, the wavelength of emitted light can also be used as an additional dimension to convey information leading to several new modulation techniques such as color shift keying (CSK) [4] and color intensity modulation (CIM) [5]. However, wavelength modulation leads to increase the complexity of the transmission and detection hardware as color light sources and multiple color lenses/filters need to be employed [6].

T-C. Bui and M. Biagi are with the Department of Information, Electrical and Telecommunication (DIET) engineering, "Sapienza" University of Rome, Rome, Italy. (e-mail: mauro.biagi@uniroma1.it).

S. Kiravittaya is with the Department of Electrical and Computer Engineering, Faculty of Engineering, Naresuan University, Phitsanulok, Thailand (email: suwitki@gmail.com).
Recently, spatial dimension that employs the spatial position of the transmitters for data modulation in a multi-input multioutput (MIMO) system has been applied for VLC system as a way to improve the spectral efficiency [7], [8]. Basically, each LED is indexed and assigned to an unique binary sequence - the spatial symbol. These symbols are then detected at the receiver by estimating the transmitted LED index based on the channel gain difference of each LED. There are various modifications based on this spatial degree of freedom. One of the first schemes is space shift keying (SSK) which allows only one light source to be active at a time period and the entire bits of a symbol is conveyed by using spatial domain. Therefore, SSK can avoid the effect of inter-channel interference and provide high data rate with low-complexity detection algorithm. Another modification is the combination of SSK and PAM and is so-called as spatial pulse amplitude modulation or spatial modulation (SM) [9]. This modulation scheme simultaneously transmits data in the signal domain by PAM and the spatial domain by SSK to achieve improved spectral efficiency. Generalized spatial modulation (GSM) can also be setup by allowing multiple active LEDs to emit PAM signal so as to outperform SSK and SM at high spectral efficiency [10]. However, both SM and GSM are affected by the nonlinearity of LEDs because of the use of PAM. In terms of power efficiency, PPM has been shown to be a favorable technique which is widely used in optical wireless communication despite of the fact that PPM provides relatively low data rate and requires both slot and symbol synchronization [11]. Spatial pulse position modulation (SPPM) is an advanced modulation technique used in optical communication that is originally proposed in [12]. This SPPM combines the classical SSK with the PPM in order to encode binary data into the spatial index of the transmit LED and the pulse position of the emitted optical intensity. Such technique can improve the energy efficiency over conventional SM.

In order to further increase the spectral efficiency while keeping low power requirement, we propose an advanced modulation technique denoted as spatial multiple pulse position modulation (SMPPM). Technically, the SMPPM can be considered as an improved version of SPPM in terms of spectral efficiency. It can be interpreted as a combination of SSK and multiple pulse position modulation (MPPM). During a symbol transmission, multiple time slots are active and only one LED out of $N_{t}$ LEDs is chosen to emit light in each active time slot. This makes SMPPM possible to modulate more signals into the positions of the multiple active time slots over the single active slot in conventional SPPM. When compared with the conventional MPPM, SSK and SPPM, the proposed 
modulation scheme achieves highest spectral efficiency. At the receiver, the optimal detector based on maximum likelihood (ML) principle is used to estimate the active transmit unit indices and active slots that can be implemented via correlator based detection. Upper bound on symbol error rate (SER) is derived and then evaluated by extensive simulations.

\section{OPTICAL SMPPM SYSTEM MODEL}

Considering a MIMO VLC system equipped $N_{t}$ transmit units (LEDs) and $N_{r}$ receive units (PDs). The symbol interval of duration $T_{s}$ is divided into $L$ time slots each having duration of $T_{p}$. In SMPPM the data symbol consists of $L_{a}$ different pulses positioned in $L$ time slots, each pulse is transmitted by an LED chosen from $N_{t}$ ones with a peak power of $P_{t}$. There can be up to $C\left(L, L_{a}\right)=L ! /\left(L_{a} !\left(L-L_{a}\right) !\right)$ possible combinations of $L_{a}$ active slots and $N_{t}^{L_{a}}$ possibilities of choosing LEDs for the above $L_{a}$ active slots. This makes SMPPM possible to encode $M_{1}=\left\lfloor\log _{2}\left(C\left(L, L_{a}\right)\right)\right\rfloor$ bits into the active pulse positions and $M_{2}=\left\lfloor L_{a} \log _{2}\left(N_{t}\right)\right\rfloor$ bits into the active LED indexes, hence resulting in a total of $M=M_{1}+M_{2}$ bits per symbol time length. The achievable spectral efficiency of SMPPM is then given by $\eta=M / L$ $\mathrm{bit} / \mathrm{s} / \mathrm{Hz}$ while the conventional SPPM and SSK are capable of transmitting $\log _{2}\left(L N_{t}\right) / L$ and $\log _{2}\left(N_{t}\right)$ bit/s/Hz, respectively. The mapping rule of SMPPM is then as follows: the incoming binary stream is first partitioned into groups of symbols each presenting $M$ bits length. During a symbol transmission, the first $M_{1}$ bits are used to determine the position of $L_{a}$ active time slots while the remaining $M_{2}$ bits in the symbol are used to indicate the index corresponding to the active LED for each active slot.

In this regard, Fig. 1 depicts the system model of SMPPM in a $4 \times 4$ MIMO VLC setup for the total time slots $L=4$ and number of active slots $L_{a}=2$. There are $C(4,2)=6$ combinations of MPPM signal and 16 combinations of active LEDs which makes SMPPM possible to transmit a number of 6 bits per symbol transmission where $M_{1}=2$ and $M_{2}=4$. The achievable spectral efficiency in this particular example is $\eta=1.5 \mathrm{bit} / \mathrm{s} / \mathrm{Hz}$. For illustration purposes, considering the symbol '001011' as in Fig. 1, the two first binary bits '00' are mapped to the first and second slots. The four remaining bits ' 1011 ' determine the indexes of the active LEDs that will be 'ON' during these two active time slots which are LED3 and LED2, respectively. Therefore, the symbol '001011' can be transmitted by using LED3 to emit light in the first time slot, and LED2 to emit light in the second time slot. At the receiver, multiple PDs are employed for receive diversity. The received signals from directed light and reflected light are converted into electrical ones by PDs and then passed through a correlator so as to proceed to detection of the transmitted signal which is estimated based on the ML criterion.

Assuming $\mathbf{x}$ is a $1 \times L$-dimensional MPPM signal vector with $L_{a}$ non-zero elements (active slots). $P$ is the average transmitted power for each symbol which is set the same for all the modulation schemes. As a result, the peak power value in each active slot for SMPPM can be calculated as $P_{t}=P L / L_{a}$. The set of positions of these $L_{a}$ nonzero
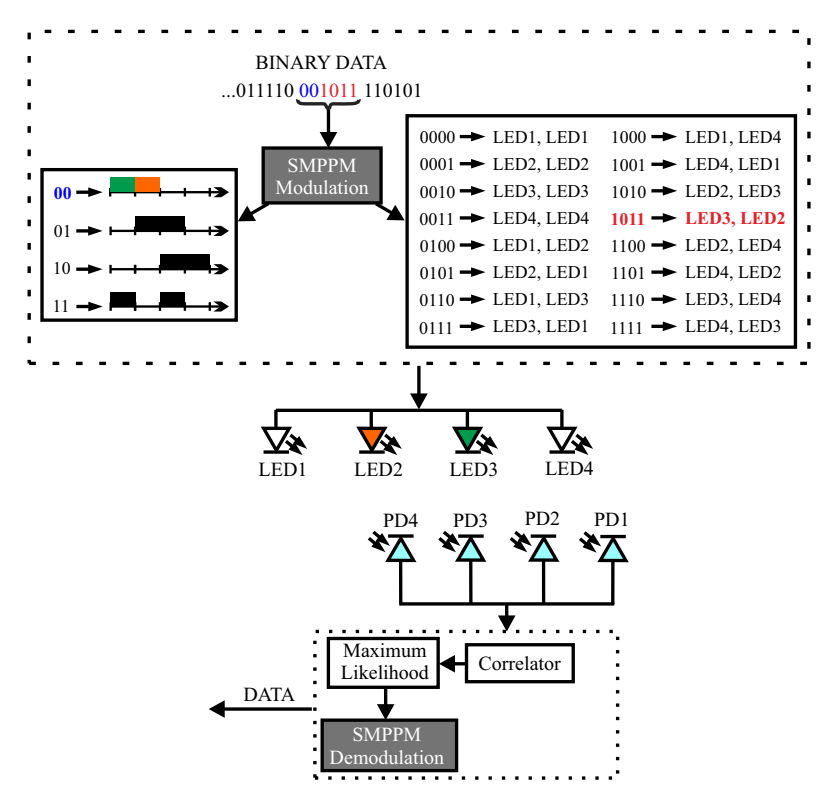

Fig. 1. System model of SMPPM in a $4 \times 4$ MIMO VLC arrangement when number of active pulses per symbol duration $L_{a}=2$ in a total number of $L=4$ slots.

elements in $\mathbf{x}$ is denoted as $\mathbf{m}=\left\{m_{1}, m_{2}, \cdots, m_{L_{a}}\right\}$. For each active slot with position $m_{i}\left(1 \leq i \leq L_{a}\right)$, it is assumed that the LED with an index $j_{i}$ is selected, this results in a set of active LED indexes that correspond to the active pulse positions set $\mathbf{m}$ as $\mathbf{j}=\left\{j_{1}, j_{2}, \cdots, j_{L_{a}}\right\}$. For example, using this principle when $\mathbf{x}=\left[0, P_{t}, P_{t}, 0\right]$ and $\mathbf{j}=\{3,1\}$ the symbol ' 010111 ' is transmitted (see the mapping table in Fig. 1).

We consider an indoor line-of-sight link configuration with multipath propagation from first reflection. The $N_{r} \times N_{t}$ channel matrix is given as follows:

$$
\mathbf{H}=\left[\mathbf{h}_{1}, \mathbf{h}_{1}, \cdots, \mathbf{h}_{N_{t}}\right]=\left[\begin{array}{cccc}
h_{11} & h_{12} & \ldots & h_{1 N_{t}} \\
h_{21} & h_{22} & \ldots & h_{2 N_{t}} \\
\vdots & \vdots & \ddots & \vdots \\
h_{N_{r} 1} & h_{N_{r} 2} & \ldots & h_{N_{r} N_{t}}
\end{array}\right]
$$

where $\mathbf{h}_{k}$ is the channel gain vector from the $k$ th LED to all the receivers, $h_{n k}$ denotes the channel gain coefficient between the $k$ th LED and the $n$th PD as discussed in detail in [13]. At the receiver, the $N_{r} \times 1$-dimensional received signal vector at $N_{r}$ receivers in the active time slot $m_{i}\left(1 \leq i \leq L_{a}\right)$ during a symbol transmission can be written as:

$$
\mathbf{y}_{m_{i}}(t)=\mathbf{s}_{m_{i}}^{\mathbf{j}, \mathbf{m}}(t)+\mathbf{n}_{m_{i}}(t), \ldots\left(m_{i}-1\right) T_{p}<t \leq m_{i} T_{p}
$$

where the notation $\mathbf{n}_{m_{i}}(t)$ is an $N_{r} \times 1$-dimensional noise vector in the $m_{i}$ time slot. It can be modeled as additive white Gaussian noise (AWGN) with zero-mean and variance $\sigma^{2}=\sigma_{\text {shot }}^{2}+\sigma_{\text {thermal }}^{2}$, where $\sigma_{\text {shot }}^{2}$ is the shot noise variance and $\sigma_{\text {thermal }}^{2}$ is thermal noise variance [13]. The term $\mathbf{s}_{m_{i}}^{\mathbf{j}, \mathbf{m}}(t)$ can be represented with the response of the channel from the $j_{i}$ LED (the active LED used in the time slot $m_{i}$ ) to all the PDs as shown in (3), where $R$ is the PD responsivity, $x\left(m_{i}\right)$ is the transmit power in the active slot $m_{i}$. 


$$
\mathbf{s}_{m_{i}}^{\mathbf{j}, \mathbf{m}}(t)=R x\left(m_{i}\right) \mathbf{h}_{j_{i}}=R P_{t} \mathbf{h}_{j_{i}}
$$

In the remaining $L-L_{a}$ time slots (inactive time slots), the received signal vectors are solely Gaussian noise samples. Consequently, one can represent the received signal at all the receivers during a symbol transmission in SMPPM by an $N_{r} \times L$ matrix $\mathbf{Y}(t)$. As in classical SPPM, correlator based detection is used in SMPPM where the received signal is passed through a bank of $L$ parallel cross-correlators with basic functions given as

$$
f_{k}(t)= \begin{cases}\frac{1}{\sqrt{T_{p}}} & \text { for }(k-1) T_{p} \leq t \leq k T_{p} \\ 0 & \text { elsewhere }\end{cases}
$$

The correlator output at all the branches of the receiver during a symbol transmission can be calculated as

$$
\mathbf{Y}=\mathbf{S}^{\mathbf{j}, \mathbf{m}}+\mathbf{N},
$$

where $\mathbf{S}^{\mathbf{j}, \mathbf{m}}$ is $N_{r} \times L$ matrix which represents for the transmitted signal when the active pulse positions set is $\mathbf{m}$ and active LED indexes is $\mathbf{j}$. There are $L_{a}$ non-zero columns in the matrix $\mathbf{S}^{\mathbf{j}, \mathbf{m}}$ that corresponds to $L_{a}$ active slots, where each nonzero column could be defined as $\mathbf{s}_{m_{i}}^{\mathbf{j}, \mathbf{m}}=\mathbf{h}_{j_{i}} \sqrt{T_{p}} R P_{t}=$ $\mathbf{h}_{j_{i}} \sqrt{E_{s} / L_{a}}$ with the symbol energy $E_{s}=L_{a}\left(R P_{t}\right)^{2} T_{p}$. Furthermore, $\mathbf{N}$ denotes the Gaussian noise in $N_{r}$ receivers for all the $L$ slots, each noise component is zero-mean uncorrelated Gaussian random variable with the same variance $\sigma^{2}=\frac{1}{2} N_{0}$, where $N_{0}$ is the noise power spectral density Assuming that the channel matrix is perfectly estimated at the receiver, the sets of active time slots and active LED indexes can be estimated using ML detection technique [14] so that it minimizes the Euclidean distance $D\left(\mathbf{Y}, \mathbf{S}^{\mathbf{j}, \mathbf{m}}\right)$ between the actual received signals at the PDs and all the possible transmitted ones and filtered by the channel as follows

$$
\begin{aligned}
{[\hat{\mathbf{m}}, \hat{\mathbf{j}}] } & =\underset{\mathbf{m}, \mathbf{j}}{\operatorname{argmin}}\left[D\left(\mathbf{Y}, \mathbf{S}^{\mathbf{j}, \mathbf{m}}\right)\right] \\
& =\underset{\mathbf{m}, \mathbf{j}}{\operatorname{argmin}} \sum_{k=1}^{L}\left(\mathbf{y}_{k}-\mathbf{s}_{k}^{\mathbf{j}, \mathbf{m}}\right)^{2}
\end{aligned}
$$

\section{SYMBOL ERROR PROBABILITY}

In this section, SER of the proposed SMPPM technique will be derived theoretically. In order to calculate the error probability, it is mathematically convenient to first derive the probability of the correct-symbol detection event. Since multiple pulses and light sources are active during a symbol transmission, decoding a correct symbol requires correctly estimating all the active light source indexes and all the active slots.

The probability of correctly decoding a SMPPM symbol becomes

$$
\begin{aligned}
P_{c}^{s y m} & =p(\hat{\mathbf{j}}=\mathbf{j} \mid \hat{\mathbf{m}}=\mathbf{m}) \times p(\hat{\mathbf{m}}=\mathbf{m}) \\
& =\prod_{i=1}^{L_{a}}\left(p\left(\hat{j_{i}}=j_{i} \mid \hat{m}_{i}=m_{i}\right) \times p\left(\hat{m}_{i}=m_{i}\right)\right) \\
& =\prod_{i=1}^{L_{a}}\left(P_{i}^{t x, c} \times p\left(\hat{m}_{i}=m_{i}\right)\right)
\end{aligned}
$$

The conditional probability, $P_{i}^{t x, c}$, of decoding a correct active LED index $j_{i}$ in SMPPM given that its active time slot $m_{i}$ is decoded correctly can be calculated similarly as the conditional probability of decoding a correct LED index in SPPM given a correct pulse position decision. In addition, since an LED is chosen independently with equally likely probability from $N_{t}$ LEDs for each active slot, $P_{i}^{t x, c}$ remains equivalent for $1 \leq i \leq L_{a}$. This probability can be then calculated by extending the derivation shown in [12] for the case of multiple receivers with the note that the symbol energy in SMPPM is now $L_{a}$ times higher than the energy in SPPM. The final expression is as follows

$$
P_{i}^{t x, c}=1-\frac{2}{N_{t}} \sum_{p=1}^{N_{t}-1} \sum_{j=p+1}^{N_{t}} Q\left(\sqrt{\frac{E_{s}}{2 L_{a} N_{0}} \sum_{k=1}^{N_{r}}\left(h_{p k}-h_{j k}\right)^{2}}\right),
$$

where $Q(x)=(1 / \sqrt{2 \pi}) \int_{x}^{+\infty} \exp \left(-t^{2} / 2\right) d t$ is the tail probability of the standard normal distribution ( $Q$ function). Accoding to this equation, the error performance is degraded when the number of active slot $L_{a}$ increases. The channel gain difference between each transmitter has a great impact on the probability of correctly decoding the active LED indexes.

On the other hand, the probability of correctly decoding each active slot is not identical for $1 \leq i \leq L_{a}$ due to the fact that choosing an active time slot can not be done independently. More specifically, the second time slot is chosen in the way so that it is not overlapped with the first active time slot, therefore the sample space is now decreased to $L-1$ slots. Similarly for the case of the $i$ th active slot, the sample space for choosing this time slot will be reduced to only $L-i$ slots. Consequently, the probability of correctly decoding an active pulse position $p\left(\hat{m}_{i}=m_{i}\right)$ in SMPPM could be calculated as:

$$
p\left(\hat{m}_{i}=m_{i}\right)=1-\frac{L-i}{N_{t}} \sum_{j=1}^{N_{t}} Q\left(\sqrt{\frac{E_{s}}{L_{a} N_{0}} \sum_{k=1}^{N_{r}}\left(h_{j k}\right)^{2}}\right) .
$$

It can be seen that the channel gain value has more influence on the active slot decision. From (7), (8), and (9), the symbol error probability of SMPPM can be derived on the basis of the union bound technique as:

$$
\begin{aligned}
& P_{e}^{s y m} \leq 1-\prod_{i=1}^{L_{a}}\left[\left(1-\frac{L-i}{N_{t}} \sum_{j=1}^{N_{t}} Q\left(\sqrt{\frac{E_{s}}{L_{a} N_{0}} \sum_{k=1}^{N_{r}}\left(h_{j k}\right)^{2}}\right)\right)\right. \\
& \left.\times\left(1-\frac{2}{N_{t}} \sum_{p=1}^{N_{t}-1} \sum_{j=p+1}^{N_{t}} Q\left(\sqrt{\frac{E_{s}}{2 L_{a} N_{0}} \sum_{k=1}^{N_{r}}\left(h_{p k}-h_{j k}\right)^{2}}\right)\right)\right]
\end{aligned}
$$

Above equation indicates that the error performance of SMPPM highly depends on the value of individual channel response as well as the correlation between themselves. Therefore, to show the effect of the channel on the performance of SMPPM we define symbol SNR as $\gamma_{s}=E_{s} / N_{0}$ which is the ratio between the transmit energy and the power spectral density of the AWGN. Using this SNR definition allows us to demonstrate error performance when taking into account individual path loss. Depending on the value of the attenuations 


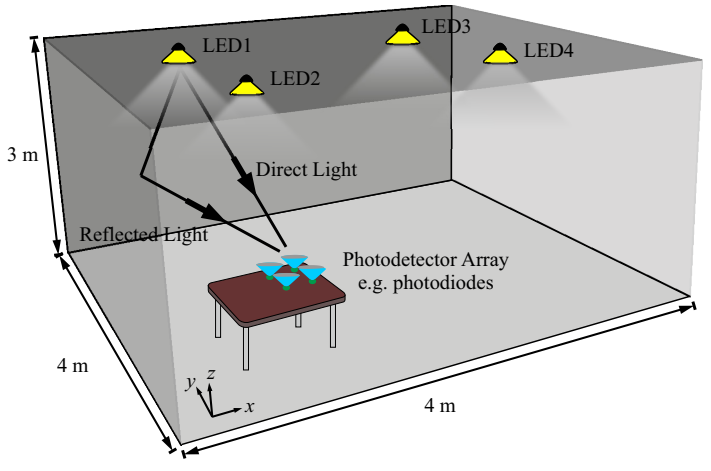

Fig. 2. Visualization of an indoor $4 \times 4$ MIMO VLC configuration with mutipath propagation from reflection. The room dimensions are $4 \mathrm{~m} \times 4 \mathrm{~m}$ $\times 3 \mathrm{~m}$, the distance from the LED plane to the receiver plane is $2 \mathrm{~m}$. The distance $d_{t x}$ between the 4 LEDs is the same and will be varied from $0.5 \mathrm{~m}$ to $2.5 \mathrm{~m}$, i.e the coordinates of the 4 LEDs when $d_{t x}=2 \mathrm{~m}$ are as follows: LED1 at $(1,3,2)$, LED2 at $(1,1,2)$, LED3 at $(3,3,2)$, and LED4 at $(3,1,2)$.

TABLE I

PARAMETERS USED IN THE SIMULATIONS

\begin{tabular}{lr}
\hline \multicolumn{2}{c}{ Room configuration } \\
\hline \multicolumn{2}{c}{ LEom dimensions } \\
Reflection coefficient of the walls \\
\hline \multicolumn{1}{c}{ LEDs } \\
\hline Number of LEDs & 4 \\
Half-power angle & $60^{\circ}$ \\
Distance from LEDs to floor & $2.5 \mathrm{~m}$ \\
Average transmitted power & $1 \mathrm{~W}$ \\
\hline \multicolumn{2}{c}{ PDs } \\
\hline Number of PDs & 4 \\
Field of view angle & $70^{\circ}$ \\
Distance from PDs to floor & $0.5 \mathrm{~m}$ \\
PD responsivity & 1 \\
\hline \hline
\end{tabular}

due to path loss, the above transmit SNR will have an offset by the average channel path loss $\alpha$ (in $\mathrm{dB}$ ) with respect to the receive $\mathrm{SNR}$.

\section{RESUlTS AND Discussions}

In the simulation, we consider an indoor $4 \times 4$ VLC scenario ( $N_{t}=4$ and $N_{r}=4$ ) with room dimension of $4 \mathrm{~m} \times 4 \mathrm{~m} \times 3$ $\mathrm{m}$ as depicted in Fig. 2. Both LEDs and PDs are placed in the middle of the room in a square $2 \times 2$ array. The transmit LEDs are directed vertically downward the floor and the receive PDs are directed vertically upward the ceiling. Separation distances between LEDs $d_{t x}$ and distances between PDs $d_{r x}$ are varied and error rate is analyzed for each case. Typical parameters of this configuration are listed in Table I. For the channel calculation, we assume all the LEDs follow the Lambertian law. Multipath propagation from light reflection is taken into account. The method for calculating channel gain coefficient with multipath propagation in this paper is adapted from [13].

Figure 3 depicts the error rate of SMPPM for both MonteCarlo simulation and analytical results derived in (10) when varying the spacings between PDs $d_{r x}$ from $0.1 \mathrm{~m}$ to $0.5 \mathrm{~m}$. The distance separation between LEDs is fixed at $2 \mathrm{~m}$. The number of active pulses is set at $L_{a}=2$ in a total of $L=4$ slots. The channel matrix for the cases of $d_{r x}=0.1,0.3$ and $0.5 \mathrm{~m}$ are shown as examples from our considered simulation

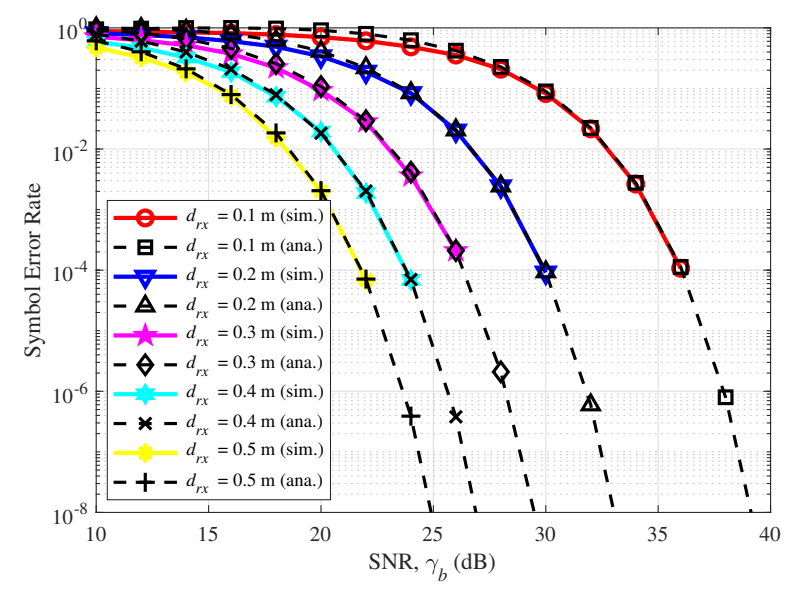

Fig. 3. Numerical results on SER of SMPPM when $L=4$ and $L_{a}=2$. The distance between LEDs is fixed at $d_{t x}=2 \mathrm{~m}$. The spacings between PDs is varied from $d_{r x}=0.1 \mathrm{~m}$ to $0.5 \mathrm{~m}$.

domain in (11) as

$$
\begin{array}{r}
\mathbf{H}_{d_{r x}=0.1 \mathrm{~m}} \approx 10^{-5}\left(\begin{array}{llll}
0.4276 & 0.4003 & 0.4003 & 0.3755 \\
0.4003 & 0.4276 & 0.3755 & 0.4003 \\
0.4003 & 0.3755 & 0.4276 & 0.4003 \\
0.3755 & 0.4003 & 0.4003 & 0.4276
\end{array}\right), \\
\mathbf{H}_{d_{r x}=0.3 \mathrm{~m}} \approx 10^{-5}\left(\begin{array}{llll}
0.4859 & 0.3959 & 0.3959 & 0.3288 \\
0.3959 & 0.4859 & 0.3288 & 0.3959 \\
0.3959 & 0.3288 & 0.4859 & 0.3959 \\
0.3288 & 0.3959 & 0.3959 & 0.4859
\end{array}\right), \\
\text { and } \mathbf{H}_{d_{r x}=0.5 \mathrm{~m}} \approx 10^{-5}\left(\begin{array}{llll}
0.6128 & 0.3731 & 0.3731 & 0.2509 \\
0.3731 & 0.6128 & 0.2509 & 0.3731 \\
0.3731 & 0.2509 & 0.6128 & 0.3731 \\
0.2509 & 0.3731 & 0.3731 & 0.6128
\end{array}\right) .
\end{array}
$$

According to (11), we have $h_{12}=h_{13}$ due to the aligned configuration of the LEDs and PDs. As a result, the channel matrix essentially consists of 3 different components which are $h_{11}, h_{12}$, and $h_{14}$ where $h_{11}>h_{12}>h_{14}$. To analyze the impact of the difference in each channel coefficient on the error performance, we define $a=h_{11}-h_{14}$ as the differential factor of the channel matrix. Therefore, channel matrix with higher $a$ value is expected to have better error performance due to the high difference between each channel coefficient.

Since the channel coefficients of the considering link are in the region of $10^{-5}$ the SER results presented in the followings will have an SNR offset of about $\alpha=100 \mathrm{~dB}$ with respect to the receive SNR [9]. For fair comparison between different link configurations and between different modulation formats, the error performances in the following are plotted against the transmit SNR per bit after removing the offset as

$$
\gamma_{b}=\gamma_{s}-10 \log _{10}(M)-\alpha
$$

It is seen from Fig. 3 that there is a well-matched agreement between simulation and theory at high SNR region. When the SNR is too low, the theoretical SER is a bit higher than the simulation due to the union bound technique [14] that has been used to approximate the SER. The error rate is rapidly 
TABLE II

DESCRIPTION OF THE CHANNEL MATRIX AT DIFFERENT $d_{t x}$

\begin{tabular}{|c|c|c|c|c|}
\hline$d_{t x}(\mathbf{m})$ & $h_{11}$ & $h_{12}$ & $h_{14}$ & $a=h_{11}-h_{14}$ \\
\hline 0.5 & $0.8138 \times 10^{-5}$ & $0.7755 \times 10^{-5}$ & $0.7398 \times 10^{-5}$ & $0.0740 \times 10^{-5}$ \\
\hline 1.0 & $0.7222 \times 10^{-5}$ & $0.6605 \times 10^{-5}$ & $0.6065 \times 10^{-5}$ & $0.1157 \times 10^{-5}$ \\
\hline 1.5 & $0.5883 \times 10^{-5}$ & $0.5228 \times 10^{-5}$ & $0.4677 \times 10^{-5}$ & $0.1206 \times 10^{-5}$ \\
\hline 2.0 & $0.4562 \times 10^{-5}$ & $0.3988 \times 10^{-5}$ & $0.3516 \times 10^{-5}$ & $0.1046 \times 10^{-5}$ \\
\hline 2.5 & $0.3485 \times 10^{-5}$ & $0.3026 \times 10^{-5}$ & $0.2654 \times 10^{-5}$ & $0.0831 \times 10^{-5}$ \\
\hline
\end{tabular}

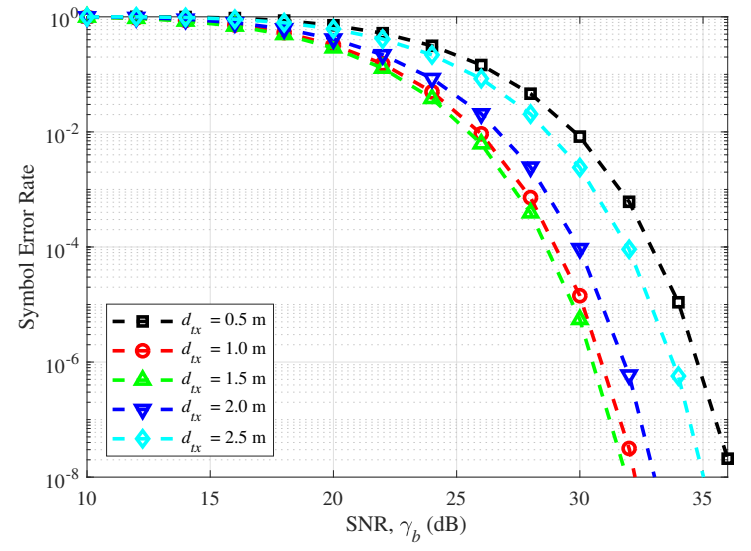

Fig. 4. SER of SMPPM when $L=4$ and $L_{a}=2$ at different LEDs separations. The distance between PDs is fixed at $d_{r x}=0.2 \mathrm{~m}$

decreased as the distance between PDs increased. At distance of $0.1 \mathrm{~m}$, achieving SER of $10^{-6}$ required SNR of about 38 $\mathrm{dB}$. However, when increasing the distance to $0.2 \mathrm{~m}$, the SNR required to get SER of $10^{-6}$ is only $32 \mathrm{~dB}$. This number keeps decreasing to only about $23 \mathrm{~dB}$ at a PD's separation of $0.5 \mathrm{~m}$. This can be explained by calculating the differential factor $a$ which is showed to continuously increase from $0.0521 \times 10^{-5}$ to $0.3619 \times 10^{-5}$ when $d_{r x}$ ranges from $0.1 \mathrm{~m}$ to $0.5 \mathrm{~m}$, respectively. Furthermore, the channel gain value at high $d_{r x}$ is also higher than at small $d_{r x}$ which increases the SNR value. This suggests that the error performance of SMPPM can be significantly improved by efficiently configuring the system so that $a$ is maximum while keeping the channel gain high.

In indoor VLC scenarios, there is a constraint in terms of illumination on the LED separation distance $d_{t x}$ since changing $d_{t x}$ results in the change of illumination level and distribution. For demonstration purpose, all the distinct values in the channel matrix and the $a$ factor are listed in Table II for different $d_{t x}$ ranging from from $0.5 \mathrm{~m}$ to $2.5 \mathrm{~m}$. With the PDs at the center, the channel gain values are high at small spacings between LEDs and vice versa, for example when $d_{t x}=0.5$ $\mathrm{m}$ the channel gain $h_{11}$ is at $0.8138 \times 10^{-5}$, however, this value is decreased to only $0.3485 \times 10^{-5}$ when increasing $d_{t x}$ to $2.5 \mathrm{~m}$. In terms of difference between each channel coefficient, increasing $d_{t x}$ gives higher $a$, however, there is a trade-off. Dealing with our considered configuration, when increasing $d_{t x}$ from $0.5 \mathrm{~m}$ to $1.5 \mathrm{~m}$, the $a$ factor increases from $0.0740 \times 10^{-5}$ to $0.1206 \times 10^{-5}$. However, at distance higher than $1.5 \mathrm{~m}, a$ is no longer increased and eventually reduced to only $0.0831 \times 10^{-5}$ at distance of $2.5 \mathrm{~m}$. The error performance of SMPPM at different LED's separation

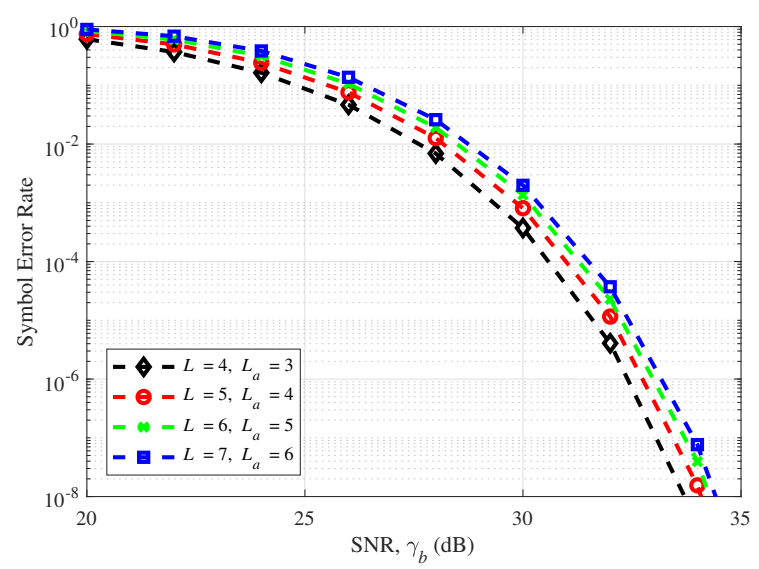

Fig. 5. Numerical results of SMPPM for the case of spectral efficiency $=2$ $\mathrm{bit} / \mathrm{s} / \mathrm{Hz}$, when varying the pulse configuration at the distance between PDs $d_{r x}=0.2 \mathrm{~m}$ and distance between LEDs $d_{t x}=2 \mathrm{~m}$.

distances is described in Fig. 4. It is seen that the SNR value required to achieve the same SER of $10^{-6}$ reduces from $35 \mathrm{~dB}$ to $30.5 \mathrm{~dB}$ when $d_{t x}$ increases from $0.5 \mathrm{~m}$ to $1.5 \mathrm{~m}$. However, when $d_{t x}$ ranges from $1.5 \mathrm{~m}$ to $2.5 \mathrm{~m}$, there is no more gain in error performance as SER is increased rapidly. This is because of the trade-off of the factor $a$ as previously discussed. In this particular system configuration, lowest SER is achieved when $d_{t x}=1.5 \mathrm{~m}$.

Since SMPPM is a pulse based modulation, the effect of the pulse configuration on the error performance should be analyzed. Figure 5 described the error probability when varying $L$ from 4 to 7 while fixing the spectral effiency at $2 \mathrm{bit} / \mathrm{s} / \mathrm{Hz}$ by varying the number of active pulses $L_{a}$. It is seen that at $\eta=2 \mathrm{bit} / \mathrm{s} / \mathrm{Hz}$, when $L$ and $L_{a}$ increase, the SNR per bit required to achieve a given probability of error is slightly increased. The SNR required when $L=7$ and $L_{a}=6$ to have error rate of $10^{-6}$ is about $33 \mathrm{~dB}$. This number is slighly reduced to about $32 \mathrm{~dB}$ when using less slots of $L=4$ and $L_{a}=3$. This is because the peak transmit power is higher when using smaller number of $L$ and $L_{a}$. Moreover, for the same spectral efficiency, the constellation size is significantly higher when more slots and pulses are employed. Therefore, at the same data rate it is preferable to employ small number of time slots to reduce the system complexity and increase the system performance. However, in some indoor systems where dimming support is required [15], this result suggests the potential dimming capability of the proposed modulation. When the peak transmit power in each active slot is fixed, changing the pulse configuration as in Fig. 5 leads to different dimming levels. It is therefore observed that SMPPM can effectively provide the same data rate at different dimming levels without severely affecting the communication link quality.

To illustrate the practicality of our scheme in indoor VLC, we plot the SER distribution of SMPPM over the considering workspace (see Fig. 2). The separation distance between PDs is $0.2 \mathrm{~m}$. The SER distribution is simulated when moving the 4 PDs to all the places in the room with resolution of $0.1 \mathrm{~m}$. The 
TABLE III

COMPARISONS OF DIFFERENT MODULATION TECHNIQUES

\begin{tabular}{|c|c|c|c|}
\hline Modulation type & Upper bound of SER & Spectral efficiency & Average power \\
\hline MPPM & $1-\prod_{i=1}^{L_{a}}\left(1-\frac{L-i}{N_{t}} \sum_{j=1}^{N_{t}} Q\left(\sqrt{\frac{\gamma_{s}}{L_{a}} \sum_{k=1}^{N_{r}}\left(h_{j k}\right)^{2}}\right)\right)$ & $\frac{\log _{2}\left(C\left(L, L_{a}\right)\right)}{L}$ & $\frac{L_{a} P_{t}}{L}$ \\
\hline SSK & $\frac{2}{N_{t}} \sum_{p=1}^{N_{t}-1} \sum_{j=p+1}^{N_{t}} Q\left(\sqrt{\frac{\gamma_{s}}{2} \sum_{k=1}^{N_{r}}\left(h_{p k}-h_{j k}\right)^{2}}\right)$ & $\log _{2}\left(N_{t}\right)$ & $P_{t}$ \\
\hline SPPM & $1-\frac{L-1}{N_{t}}\left(1-\frac{2}{N_{t}} \sum_{p=1}^{N_{t}-1} \sum_{j=p+1}^{N_{t}} Q\left(\sqrt{\frac{\gamma_{s}}{2} \sum_{k=1}^{N_{r}}\left(h_{p k}-h_{j k}\right)^{2}}\right)\right)$ & $\frac{P_{t}}{L}$ \\
\hline SMPPM & $\times \sum_{l=1}^{N_{t}}\left(\frac{1}{L-1}-Q\left(\sqrt{\gamma_{s} \sum_{k=1}^{N_{r}}\left(h_{l k}\right)^{2}}\right)\right)$ & $\frac{1}{L} \log _{2}\left(N_{t} L\right)$ & $\frac{\log _{2}\left(C\left(L, L_{a}\right)\left(N_{t}^{L_{a}}\right)\right)}{L}$ \\
\hline
\end{tabular}

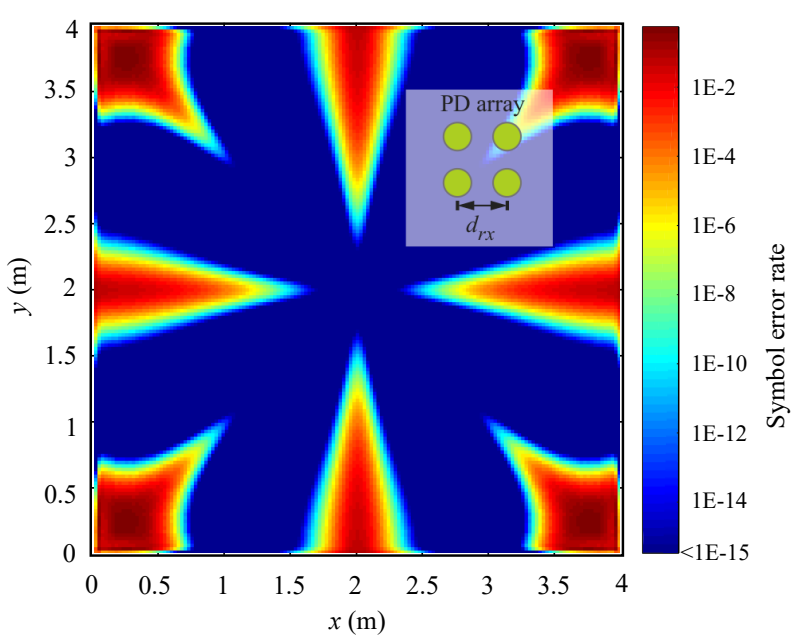

Fig. 6. The SER distribution of SMPPM in the proposed indoor VLC system with multipath propagation from reflection. The PD separation is $d_{r x}=0.2$ m.

channel matrix with reflection and transmit SNR are calculated in every position of PDs and SER is then simulated as shown in Fig. 6. According to that, the places under the middle and cross lines in the room have very high error compared with the other positions due to the effect of high channel correlation around these lines. At regions around the corners, the SER increases rapidly and stand at more than $10^{-3}$. This is because the channel response values from the LEDs to PDs in these regions are small in conjunction with the high correlation between them. In the other regions far from the middle and cross lines, the SER is extremely small. The error can even be lower than $10^{-15}$ in many places. In general, SMPPM shows favorable error performance in most of the places in an indoor VLC configuration.

The expressions for comparison of all previously mentioned schemes in terms of SER, average transmitted optical power per symbol duration, and spectral efficiency are shown in Table III. In this table, the peak transmitted power $P_{t}$ of an LED in each slot is the same for all the scheme in order to calculate the average transmit power for every symbol. It is seen that
SPPM shows best performance in terms of power efficiency as since it requires power of only $P_{t} / L$ watt per symbol. SMPPM and MPPM both have the same transmitted power that is $L_{a} P_{t} / L$ watt, while SSK consumes an average power of $P_{t}$ watt per symbol. However, in indoor VLC, there is less constraint on the average optical power due to the fact that LEDs are used both for illumination and communication purposes [15]. Therefore, varying transmitted power in indoor VLC is a topic of dimming and will be considered in our future work.

In terms of SER, Fig. 7 shows performance of SMPPM and conventional techniques, SPPM and SSK at the same average transmit power $P$. According to Fig. 7, SPPM shows best error performance ranging from 1.8 and $4 \mathrm{~dB}$ better than SMPPM (at $L_{a}=3$ ) and SSK respectively. This is, however, at the expense of lowest achievable spectral efficiency of only $\eta=0.625 \mathrm{bit} / \mathrm{s} / \mathrm{Hz}$. In the meantime, SMPPM with $L_{a}=7$ has highest spectral efficiency of $\eta=2.125 \mathrm{bit} / \mathrm{s} / \mathrm{Hz}$ and gives better error performance than SSK at high SNR regime. The spectral efficiency of SMPPM at $L_{a}=3$ is $1.375 \mathrm{bit} / \mathrm{s} / \mathrm{Hz}$ with SER of about $2.2 \mathrm{~dB}$ better than in SSK. Therefore, when the system requires the balance between achievable data rate and error rate, SMPPM with $L_{a}=3$ can be utilized in this particular configuration.

Overall, SMPPM gives much higher spectral efficiency value than in MPPM, SSK and SPPM as presented in Fig. 8. For the case of 4 light sources, the spectral efficiency of SSK is $2 \mathrm{bit} / \mathrm{s} / \mathrm{Hz}$. When 32 pulse slots $(L=32)$ are used, SPPM provides a spectral efficiency of nearly 0.5 . On the other hand, when more pulses are active (SMPPM) the spectral efficiency is significantly increased and can be larger than those in SSK when the number of active pulses in the system increases and approach the total pulse $L$. The maximum spectral efficiency of SMPPM in this four-transmitters configuration can be up to about $2.25 \mathrm{bit} / \mathrm{s} / \mathrm{Hz}$ when the number of active pulse slots $L_{a}$ approaches 27. For the case of MPPM, since the information is modulated solely on the active pulse positions, its spectral efficiency is peak at below $1 \mathrm{bit} / \mathrm{s} / \mathrm{Hz}$ at the middle range of the number of active pulse slots and reduced gradually on the two sides. Besides, the number of light sources in 


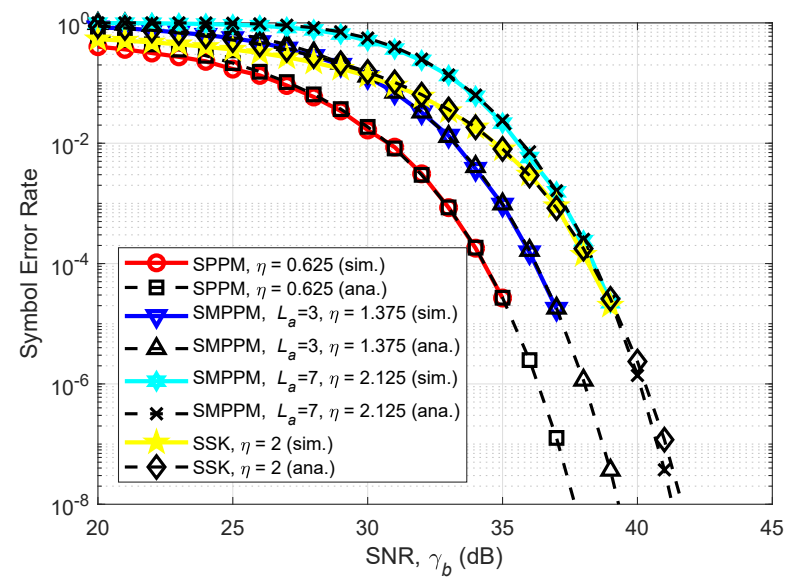

Fig. 7. SER of SMPPM compared with SPPM and SSK in the proposed configuration at different spectral efficiencies $\eta \mathrm{bit} / \mathrm{s} / \mathrm{Hz}$. The PD separation is $d_{r x}=0.2 \mathrm{~m}$. The LED separation is $d_{t x}=2 \mathrm{~m}$. The total number of time slots per symbol duration is $L=8$ and the number of active slots $L_{a}$ is varied.

SMPPM is not restricted to be the power of two as in SSK and SPPM. This gives the flexibility in designing of the indoor optical wireless communication systems in order to meet other requirements such as illumination level and coverage area of the light sources.

\section{CONCLUSiON}

In this paper, a multi-pulse multi-transmitter modulation technique called spatial multiple pulse position modulation is proposed and analyzed by combining SSK with MPPM to form a high efficient modulation scheme for VLC. SMPPM gives highest spectral efficiency when compared with conventional MPPM, SPPM and even higher than in SSK for high number of active slots. The error probability of SMPPM in a MIMO system is derived theoretically using union bound technique and is then verified by means of Monte-Carlo simulations. In terms of SER, SMPPM has lower error rate than in SSK at high SNR regime with higher spectral efficiency. SPPM shows best performance in terms of error rate at the expense of lowest spectral efficiency. The effect of individual channel gain values and the difference between them on the error performance of SMPPM is also demonstrated. Simulation results show that there is a trade-off when increasing the transmitter's separation distance on the error performance of SMPPM. The error rate can be significantly reduced by properly configuring the LEDs, PDs and the modulating signal. SMPPM therefore can be utilized to provide the balance between error rate and data rate with low complexity of the whole VLC system.

\section{ACKNOWLEDGMENT}

This research was financially supported by the Graduate School and Faculty of Engineering of Naresuan University and Sapienza University of Rome. The authors would like to acknowledge the Naresuan University International Student Scholarships (the Academic Year 2015).

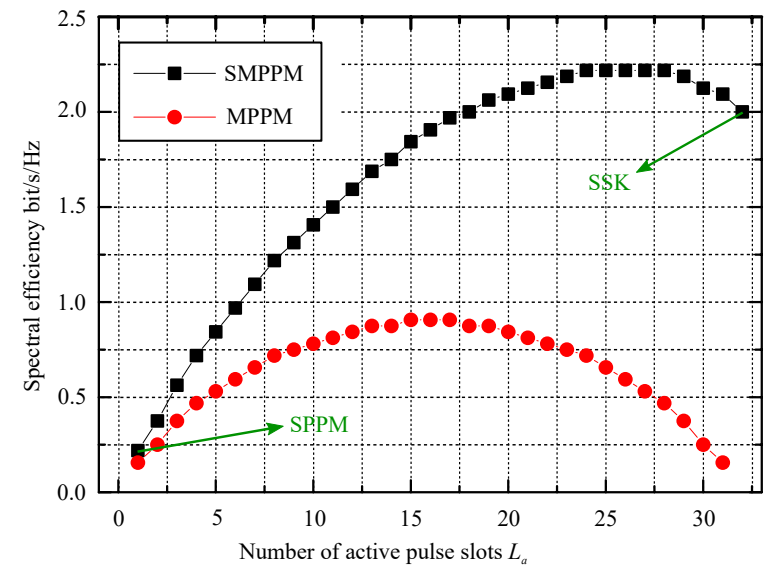

Fig. 8. Comparison of SMPPM, SSK, SPPM and MPPM in terms of spectral efficiency when varying the number of active pulses in a total of 32 slots $(L=32)$.

\section{REFERENCES}

[1] T. Komine and M. Nakagawa, "Fundamental analysis for visible-light communication system using led lights," IEEE Transactions on Consumer Electronics, vol. 50, no. 1, pp. 100-107, Feb 2004.

[2] D. Karunatilaka, F. Zafar, V. Kalavally, and R. Parthiban, "Led based indoor visible light communications: State of the art," IEEE Communications Surveys Tutorials, vol. 17, no. 3, pp. 1649-1678, thirdquarter 2015.

[3] Z. Ghassemlooy, W. Popoola, and S. Rajbhandari, Optical Wireless Communications: System and Channel Modelling with MATLAB. CRC Press, Inc., 2012.

[4] J. Luna-Rivera, R. Perez-Jimenez, J. Rabadan-Borjes, J. Rufo-Torres, V. Guerra, and C. Suarez-Rodriguez, "Multiuser csk scheme for indoor visible light communications," Optics Express, vol. 22, no. 20, pp. 24 256-24 267, Oct 2014.

[5] K. I. Ahn and J. K. Kwon, "Color intensity modulation for multicolored visible light communications," IEEE Photonics Technology Letters, vol. 24, no. 24, pp. 2254-2257, Dec 2012.

[6] S. Pergoloni, A. Petroni, T.-C. Bui, G. Scarano, R. Cusani, and M. Biagi, "Ask-based spatial multiplexing rgb scheme using symbol-dependent self-interference for detection," Opt. Express, vol. 25, no. 13, pp. 15028 15042 , Jun 2017.

[7] T. Fath, M. D. Renzo, and H. Haas, "On the performance of space shift keying for optical wireless communications," in 2010 IEEE Globecom Workshops, Dec 2010, pp. 990-994.

[8] R. Mesleh, H. Elgala, and H. Haas, "Optical spatial modulation," IEEE/OSA Journal of Optical Communications and Networking, vol. 3 , no. 3, pp. 234-244, March 2011.

[9] T. Fath and H. Haas, "Performance comparison of mimo techniques for optical wireless communications in indoor environments," IEEE Transactions on Communications, vol. 61, no. 2, pp. 733-742, February 2013.

[10] S. P. Alaka, T. L. Narasimhan, and A. Chockalingam, "Generalized spatial modulation in indoor wireless visible light communication," CoRR, vol. abs/1503.03308, 2015.

[11] M. Biagi, A. M. Vegni, S. Pergoloni, P. M. Butala, and T. D. C. Little, "Trace-orthogonal ppm-space time block coding under rate constraints for visible light communication," Journal of Lightwave Technology, vol. 33, no. 2, pp. 481-494, Jan 2015.

[12] W. O. Popoola, E. Poves, and H. Haas, "Spatial pulse position modulation for optical communications," Journal of Lightwave Technology, vol. 30, no. 18, pp. 2948-2954, Sept 2012.

[13] J. M. Kahn and J. R. Barry, "Wireless infrared communications," Proceedings of the IEEE, vol. 85, no. 2, pp. 265-298, Feb 1997.

[14] J. G. Proakis, Digital Communications. McGraw-Hill, 2000.

[15] F. Zafar, D. Karunatilaka, and R. Parthiban, "Dimming schemes for visible light communication: the state of research," IEEE Wireless Communications, vol. 22, no. 2, pp. 29-35, April 2015 